\title{
Therapeutic Potential of Metformin and Vitex Agnus-Castus In Alleviating Cardiac Damage Induced By Hyperandrogenism In Polycystic Ovary Female Rats
}

Widad M. Al-Bishri

Biochemistry Department, Faculty of Science-Al Faisaliah, King Abdulaziz University, Jeddah, KSA

Address For Correspondence:

Widad M. Al-Bishri, Biochemistry Department, Faculty of Science- Al Faisaliah, King Abdulaziz University, Jeddah, KSA.

E-mail: walbishry@kau.edu.sa

\section{A RTICLE INFO}

\section{Article history:}

Received 12 October 2017

Accepted 22 November 2017

Available online 6 December 2017

Keywords:

Polycystic Ovarian Syndrome, Vitex agnus-castus, hyperandrogenism, hyperleptinemia

hyperprolactinemia

\begin{abstract}
A B S T R A C T
This research was aimed at exploring the profound therapeutic impacts of metformin (Met) and Vitex agnus-castus fruit (Vit) on cardiovascular risk factors, which includes dyslipidaemia, hyperhomocysteinemia and hyperleptinemia as well as hypoadiponectinemia. Others are hyperprolactinemia as well as DNA oxidative damage. These factors are generated by hyperandrogenism in Poly-Cystic Ovarian Syndrome (PCOS) female rats. The task involved inducing PCOS in rats using an oral ingestion of letrozole of $1 \mathrm{mg} / \mathrm{kg}$ once a day for twenty-one days. Rats, which were induced with PCOS, were treated orally with either Met of $70 \mathrm{mg} / \mathrm{kg}$ single dose or Vit fruit amounting to $500 \mathrm{mg} / \mathrm{kg}$ daily for 21 days after PCO induction. The findings indicate that giving rats induced with PCOS Met or Vit significantly ameliorated the increases in hyperandrogenism markers, including androstendione, Dehydroepiandrosterone Sulfate (DHEAS) and total testosterone as well as free testosterone in comparison with PCOS untreated ones. The two agents did modulation of the reduction in the content of serum of apolipoprotein (apo) A. Additionally; they elevated total lipid as well as apo B of rats with PCOS. Furthermore, the findings showed Met or Vit significantly ameliorating the serum hyperhomocysteinemia, hyperleptinemia, hypoadiponectinemia and hyperprolactinemia. There was also the increase in 8-hydroxydeoxyguanosine (8-OHdG) in PCOS rats, when compared to PCOS untreated rats. It became evident that Met and Vit could modulate the increases in the serum cardiac damage markers such as troponine $\mathrm{T}$ and alkaline phosphatase (ALP) as well as lactate dehydrogenase (LDH) in the PCOS induced rats. Finally, it became clear through the findings that treating PCOS rats using Vit could yield more efficient results in modulation of most the studied markers compared to Met. Conclusion: Met or Vit can be used to reduce cardiovascular risk. This is as a result of Met and Vit effective showing the ability that they can modulate cardiovascular risk elements in the PCOS induced rats utilised. However; Vit proved to be more potent than Met as far as this therapy went and this explained why Vit fruit is used in managing PCOS related cardiovascular events.
\end{abstract}

\section{INTRODUCTION}

It is public knowledge that Polycystic Ovarian Syndrome (PCOS) has become a prevalent endocrine heterogeneous disorder for the female gender. Polycystic Ovarian Syndrome is also regarded as the most common cause of hyperprolactinemia which, is an ovulatory infertility that affects $5 \%$ to $10 \%$ women within their reproductive age (Taher \& Atia, 2012; Yilmaz et al., 2015). Ehrmann (2005) reiterates that it is the most dangerous factor that causes the development of numerous diseases including diabetes and cardiovascular disease (CVD). Hyperandrogenism, also known as androgen excess, is the common pathophysiological hormonal marker of PCOS. In the view of Kamel (2005), it is characterised by excessive levels of male sex

Open Access Journal

Published BY AENSI Publication

(C) 2017AENSI Publisher All rights reserved

This work is licensed under the Creative Commons Attribution International License (CC BY).

http://creativecommons.org/licenses/by/4.0/

\section{(c) (i) Open Access}

To Cite This Article: Widad M. Al-Bishri Therapeutic potential of metformin and Vitex agnus-castus in alleviating cardiac damage induced by hyperandrogenism in polycystic ovary female rats Aust. J. Basic \& Appl. Sci., 11(14): 129-138, 2017 
hormones that include testosterone (androgens). Ovaries as well as adrenal cortex have both contributed to hyperandrogenism in women having PCOS (Kamel, 2005). This implies that increased ovarian androgen production is the major contributor to androgen excess in women patients with PCOS (Baptiste et al., 2010). However, approximately $20 \%$ to $50 \%$ of women with classic ovulatory PCOS experience adrenal androgen excess. Baptiste et al. (2010) notes that this is shown when dehydroepiandrosterone sulfate (DHEAS) increases in level and which gets secreted by adrenal cortex. The latter steroid's role is equally significant especially in the androgen hormones biosynthesis (Endoh et al., 1996). According to Lizneva et al. (2016), PCOS has clinical symptoms that include hirsutism and acne resulting from increased androgen secretion.

Many reports revealed that PCOS is associated with many metabolic abnormalities. These metabolic abnormalities include dyslipidaemia (Valkenburg et al., 2008), hyperhomocysteinemia (Esmaeilzadeh et al., 2017), hyperleptinemia, hypoadiponectinemia, hyperprolactinemia and hyperinsulinemia (Valkenburg et al., 2008; Henry, 2011; Chen et al., 2015), which are important hazard elements for cardiovascular diseases. Even though people might realise the presence of cardiovascular risk factors in adulthood, these factors may appear at an early age. Women are more prone to be affected while young than men are.

Managing PCOS with insulin lower agents that include metformin or herbal medicine such as Vitex agnuscastus have been documented. Ren et al. (2001) has shown that herbal medicines contain pharmacologically active constituents which are capable of producing physiological effects on female endocrinology. The researchers add that these constituents can be linked positively to reduced CVD incidences.

Moghetti et al. (2000) argues that it is common knowledge that Metformin (Met), as an insulin-sensitizing drug, is employed when managing type 2 diabetes. It is, nonetheless, worth noting that it suppresses the ovarian cytochrome p450c17 activity. In the view of Moghetti et al. (2000), it also has the capability of ameliorating hyperandrogenism as well as normalising ovulation in women having PCOS. Moreover, it was established after a clinical enquiry that Met is capable of reducing significantly the levels of prolactin, enhance index of free androgen. Ibanez et al. (2000) and Elsersy (2017) note that it can also enhance levels of blood total testosterone and androstenedione as well as DHEAS. Additionally, Met increases glucose uptake through adipocytes and muscle cells as well as enhances disposal of insulin-stimulated glucose (Reaven et al., 1992).

On the other hand; Vitex agnus-castus (Vit), (also referred to as chaste-tree berry or family verbenaceae) describes a traditional plant often used when treating acne, digestive complaints, hyperprolactinemia and menstrual irregularities as well as infertility in women (Arentz et al., 2014; Elsersy, 2017). According to scholars such as Sliutz et al. (1993) and Arentz et al. (2014), Vit has various forms of phyto compounds binding to the receptors of dopamine type 2 (DA-2) situated in the brain. Such compounds minimise cyclic adenosine mono phosphate (cAMP) as well as the prolactin's depleted secretion. As aforementioned, Vit lowers the levels of prolactin and this discovery was made in normal as well as ovariectomised rats (Ibrahim et al., 2008). It therefore became evident that Vit extracts have numerous active compounds including flavonoids, essential oils and diterpenes as well as glycosides (Weiss, 1988). Furthermore, the diterpenes display activity of dopaminergic in cultured pituitary cells. This gives rise to prolactin production's suppression (Wuttke et al., 2003). Wuttke et al. (2003) adds that it is also good to note that the glycosides can impact indirectly on hormones on in-vitro clinical trials. Apigenin, as well, exhibits regulatory effects on homeostasis of fat tissue (Wuttke et al., 2003).

It is no secret that Vit fruit has often been utilised when treating reproductive disorders in patients having PCOS, but is important to note that this fruit's exact mechanisms in the management of PCOS as well as its cardiovascular complications are not established yet. Therefore, in this case, the researcher mainly aimed at highlighting the profound therapeutic impacts of Vitex agnus-castus fruit as compared to the available drug in the name of metformin with regard to controlling hyperandrogenism and related cardiovascular risk factors such as dyslipidaemia, hyperhomocysteinemia, hyperleptinemia, hypoadiponectinemia and hyperprolactinemia as well as DNA oxidative damage in polycystic ovary syndrome induced female rats.

\section{MATERIALS AND METHODS}

\section{Chemicals:}

Every chemical utilised in this study was high in purity. The chemicals were supplied by Sigma and Merck companies. Metformin hydrochloride was acquired from Merck, a company located in Germany.

\section{Plants:}

This was majorly Vitex agnus-castus, which was bought from General Nutrition Center (GNC) in its powder form.

\section{Animals:}

The investigation used 40 adult female Wistar Albino rats that weighed between $180 \mathrm{~g}$ and 200g. Animal House Colony of King Fahad Medical Research Center in Jeddah supplied the rats. They were given two weeks to adapt. The investigator kept these rats within standard polypropylene cages with a controlled environment of 
$25^{\circ} \mathrm{C} \pm 2$ and $12 \mathrm{~h}$ light/dark cycle as well as $50 \%$ humidity. They were allowed access to food and water. Since the validity of this experiment had to be beyond reproach, the researcher invited the Ethical Committee of King Fahad Medical Research Center. Jeddah, KSA to approve it.

\section{Experimental Design:}

Rats were categorised into four groups, with ten rats in every group:

Group 1: Healthy control.

Group 2: Polycystic Ovary syndrome (PCOS): Oral means was utilised in administering letrozole to induce PCOS in the rats. Dosage was $(1 \mathrm{mg} / \mathrm{kg}$ ) for a twenty-one day period (Reddy et al., 2016).

Group 3: PCO induced rats given single dose oral treatment of $70 \mathrm{mg} / \mathrm{kg}$ using metformin (Met) after the induction of PCO (Lal \& Jain, 2010).

Group 4: PCO induced rats treated orally with powder of Vitex agnus-castus (Vit) fruits (500 mg/kg) for a fifteen-day period after PCO induction (Allahtavakoli et al., 2015).

The investigator dissolved Letrozole in $0.5 \%$ carboxy methyl cellulose (CMC), the Vitex agnus-castus fruit powder and metformin hydrochloride were put in a distilled water to dissolve. It is also important to note that control rats have orally ingested vehicle of $0.5 \% \mathrm{CMC}$ on a daily basis throughout the period of the experiment.

Close to the termination of this experiment that took 5 weeks, the investigator decided to fast animals overnight as well as gather blood specimens, after which the serum was separated through centrifugation and utilised varyingly in biochemical analysis

\section{Serum Biochemical Analysis:}

Radioimmunoassay method was applied in determining androstenedione (Coyotupa et al., 1973). Dehydroepiandrosterone sulphate (DHEAS) was estimated by use of competitive rat DHEAS ELISA kit (MyBioSource, Inc., USA). A competitive rat testosterone ELISA kit was used in measuring total testosterone (Catalog Number, 55-TESMS-E01; Alpco, Salem, NH, USA). For the estimation of free testosterone, a colorimetric competitive rat testosterone ELISA kit was used (BioVendor, Laboratorni medicina a.s. Czech Republic).When it came to total lipids, an enzymatic calorimetric assay, uautoanalyzer was utilised (Mindray BS-Clinical Chemistry Analyzer, Guangzhou Shihai Medical Equipment Co, Guangdong, China). Then the Apolipoprotein (apo) A as well as apo B concentrations were established using nephelometric assay and the Mono Binding kit (Binding Site, Birmingham, UK) in conformity to the manufacturer's instructions. A commercial rat ELISA kit helped in estimating leptin (catalog number MOB00; R\&D Systems, Minneapolis, MN, USA). The one used for adiponectin rat ELISA kit (catalog number 44-ADPRT-E01; Alpco, Salem, NH, USA), Homocysteine's was radioenzymatic assay which depended on conversion of homocysteine to Sadenosylhomocysteine in presence of adenosine as well as S-adenosylhomocysteine hydrolase (Refsum et al., 1985). Evaluating prolactin, on the other hand, required sandwich rat prolactin ELISA kit (Cat. No. KT-583, Kamiya Biomedical Company, 12779 Gateway Drive Seattle, WA 98168 U.S.A). 8-OH-dG content of serum was measured using high-performance liquid chromatography having sytems of electrochemical detection (HPLC-ECD systems), as aforementioned (Nakae et al., 1995). Troponin T estimation was done by a Siemens Dimension Xpand Plus instrument (IL, USA), and the one for ALP and LDH, an autoanalyzer (Abbott Laboratories, USA).

\section{Statistical Analysis:}

The results were expressed in the form of mean \pm standard error (SE). The number of animals were ten. The experiment used ANOVA and Bonferroni's test to analyse significant variations. Significance level was $\mathrm{P}<0.05$.

\section{Results:}

Figure 1 shows impacts of Met and Vit on serum hyperandrogenism indices. These include, for example, androstendione, dehydroepiandrosterone sulfate (DHEAS), total testosterone and free testosterone in female rats having PCOS. According to the data, markers in female rats with PCOS (group 2) increase compared to controlled ones (group 1, P $\leq 0.001$ ). Met or Vit fruits' oral administration to rats induced with PCOS (groups 3 $\& 4$ respectively), showed hyperandrogenism indices reduced levels when with regard to PCOS untreated animals $(P \leq 0.001)$. Vit fruit significantly proved effective in modulating these parameters against Met treated ones.

Therefore, PCOS induction in female rats yielded hyperlipidemia (Figure 2) through significant serum content reduction of apolipoprotein (apo) A and total lipid and apo B concentration elevation with regard to control animals $(P \leq 0.001)$. Met or Vit treatment saw down-regulations of deviations regarding these indices against PCOS untreated animals $(P \leq 0.001)$. Vit fruit reduced hyperlipidemia parameters in PCOS rats more successfully than Met treated ones $(P \leq 0.05)$.

In Figure 3, Met and Vit fruit impacts on the serum homocysteine (Hcy) and adepokines (leptin and adeponectin) in PCOS induced rats are depicted. It is observed that rats having PCOS bear significant 
hyperhomocysteinemia, hyperleptinemia and hypoadiponectinemia compared to control ones $(P \leq 0.05)$. Met or Vit taken orally, markedly modulated the parameters deviations in rats with PCOS compared to PCOS untreated rats $(P \leq 0.001)$.

Moreover, experiment demonstrated that PCOS induction in rats, brought significant serum content increases concerning prolactin and 8-hydroxydeoxyguanosine (8-OHdG, an index of oxidative DNA damage ) versus control animals $(P \leq 0.001$, Figure 4$)$. Met or Vit ingestion minimised markedly the index concentrations in rats with PCOS versus PCOS untreated rats $(P \leq 0.001)$. Results indicated that Vit reduced prolactin effectively.

In Table 1, there are the serum markers of cardiac function including troponine T, ALP and LDH placed variedly in groups of PCOS rats. The data illustrated significant elevation in rats with PCOS versus the control rats $(P \leq 0.001)$. Furthermore, Met or Vit normalized such markers particularly PCOS untreated rats $(P \leq 0.001)$. It is reiterated that Vit fruit can reduce these indices to normal levels.
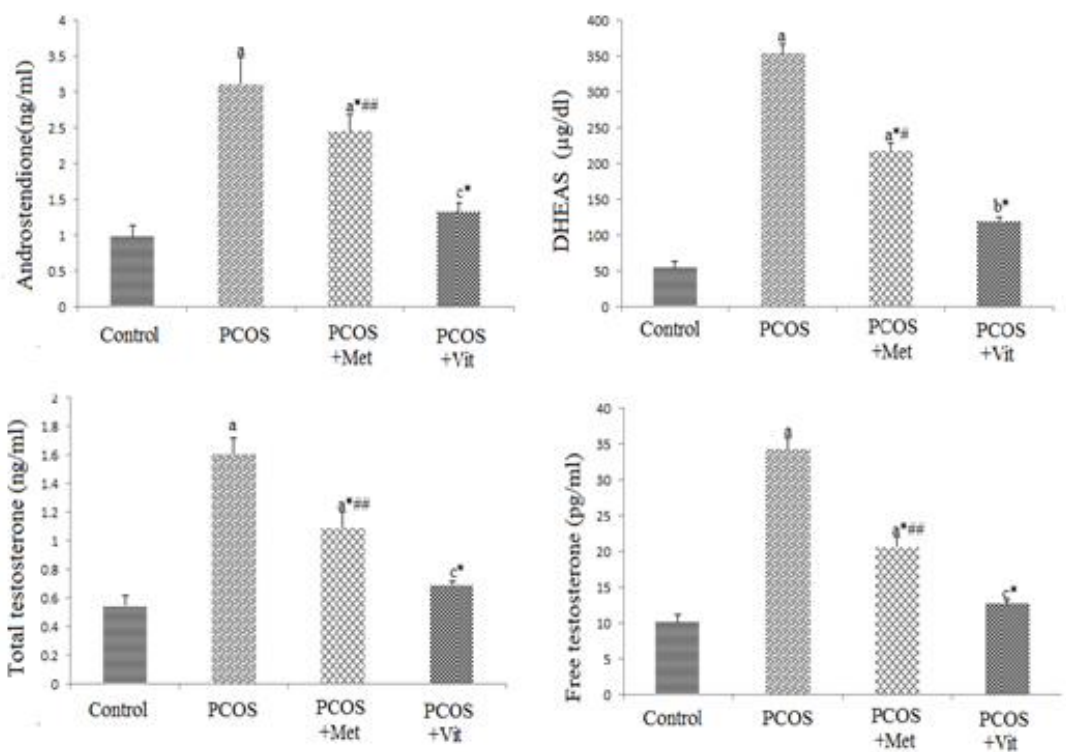

Fig. 1: Effect of metfomin (Met) and Vitex agnus-castus fnit (Vit) on senm hyperandrogenism markers (androstendione, dehydroepiandrosterone sulfate (DHEAS), total testosterone and free testosterone) in female rats with PCOS. Values are calculatd as mean \pm S.E $(\mathrm{n}=10),{ }^{a} P \leq 0.001,{ }^{b} P \leq 0.01,{ }^{c} P \leq 0.05$ in comparison with the control group, ${ }^{*} P \leq 0.001$ with respect to PCOS rats, ${ }^{*} P \leq 0.05,{ }^{* *} P \leq 0.01$ with respect to PCOS rat group treated with Vit.
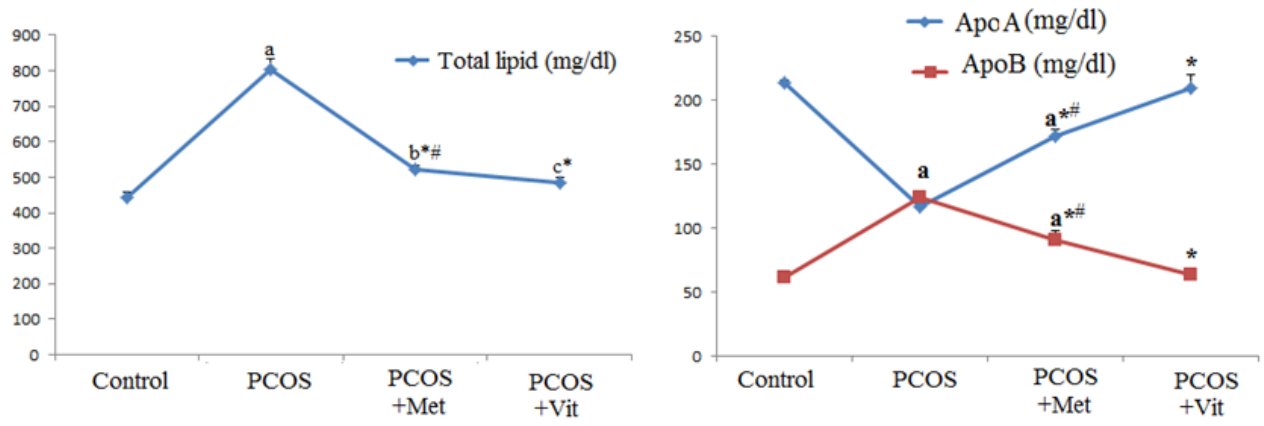

Fig. 2: Effect of metfomin (Met) and Vitex agnus-castus fnit (Vit) on senum total lipid, apolipoprotein A (Apo A) and apolipoprotein B (Apo B) in female rats with PCOS. Values are calculatd as mean \pm S.E $(n=10)$, ${ }^{a} P \leq 0.001,{ }^{b} P \leq 0.01,{ }^{c} P \leq 0.001$ in comparison with the control group, ${ }^{*} P \leq 0.001$ with respect to PCOS rats, ${ }^{*} P \leq 0.05,{ }^{* *} P \leq 0.01$ with respect to PCOS rat group treated with Vit. 

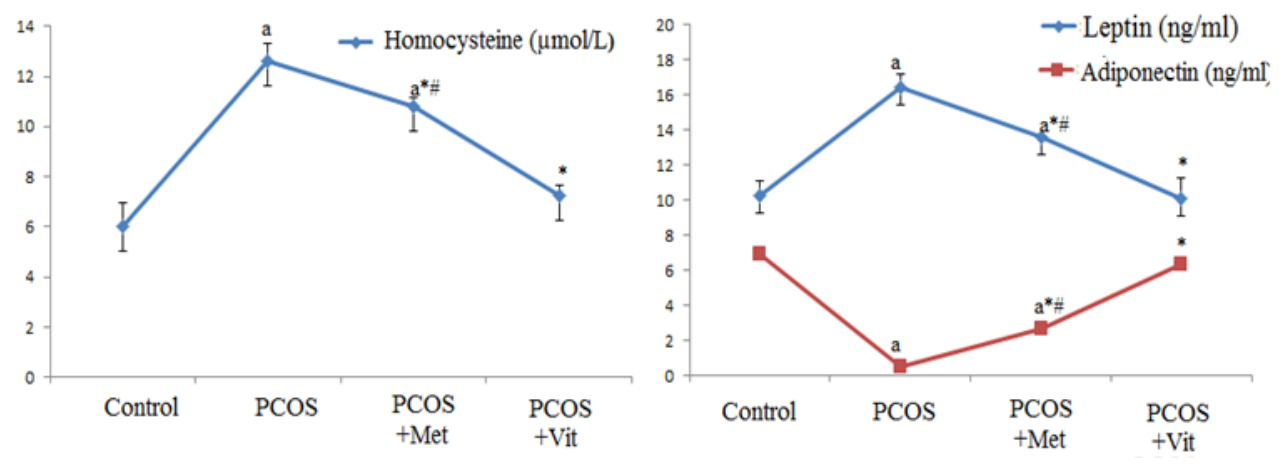

Fig. 3: Effect of metfomin (Met) and Vitex agnus-castus fnit (Vit) on senum homocysteine and adipokines (Leptin and adeponectin) in female rats with PCOS. Values are calculatd as mean \pm S.E $(n=10)$, ${ }^{a} P \leq 0.001$, in comparison with the control group, ${ }^{*} P \leq 0.001$ with respect to PCOS rats, ${ }^{*} P \leq 0.05$, with respect to PCOS rat group treated with Vit.
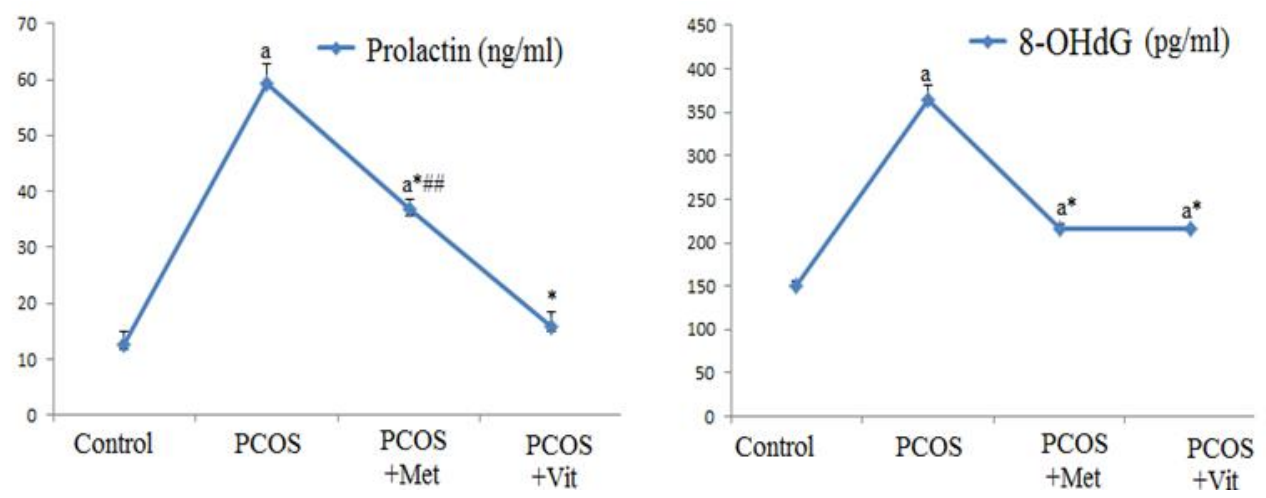

Fig. 4: Effect of metfomin (Met) and Vitex agnus-castus fnit (Vit) on senum prolacin and 8hydroxydeoxyguanosine $(8-\mathrm{OHdG})$ in female rats with PCOS. Values are calculatd as mean \pm S.E $(\mathrm{n}=10),{ }^{a} P \leq 0.001$, in comparison with the control group, ${ }^{*} P \leq 0.001$ with respect to PCOS rats, ${ }^{* *} P \leq 0.01$, with respect to PCOS rat group treated with Vit.

Table 1: Met and Vit's effects on indices of serum cardiac function in PCOS induced female rats

\begin{tabular}{|l|l|l|l|l|}
\hline PCO+Metformin & PCOS +Vit & Control & PCO & Parameters \\
\hline $84.33 \pm 2.5^{\mathrm{b}^{* \#}}$ & $60.2 \pm 2.34^{*}$ & $61.66 \pm 7.7$ & $128.66 \pm 6.5^{\mathrm{a}}$ & ALP $(\mathrm{U} / \mathrm{L})$ \\
\hline $12.5 \pm .98^{*}$ & $10.65 \pm 1.62^{*}$ & $10.83 \pm 1.45$ & $20.98 \pm 1.64^{\mathrm{a}}$ & Troponine T(pg/ml) \\
\hline $336.56 \pm 27.6^{\mathrm{a}^{*} \#}$ & $224.66 \pm 5.68^{*}$ & $230.36 \pm 4.35$ & $570.45 \pm 50.00^{\mathrm{a}}$ & $\mathrm{LDH}(\mathrm{U} / \mathrm{L})$ \\
\hline
\end{tabular}

Values are calculated as mean \pm S.E. $(\mathrm{n}=10),{ }^{a} P \leq 0.001,{ }^{b} P \leq 0.01$ in comparison with the control group, ${ }^{*} P \leq 0.001$ with respect to PCOS rats, ${ }^{\#} P \leq 0.05,{ }^{\#} P \leq 0.01$ with respect to PCOS rat group treated with Vit.

\section{Discussion:}

Recent researches evidences indicate that PCOS can be linked to both female reproductive disorder and a leading cause of CVD (Yarali et al., 2001). The traditional drugs are not adequately utilised because they yield dangerous side-effects and are ineffective. Jang et al.(2014) notes that this calls for alternative therapy. They give an example of using natural products that have multifunctional impacts.

This experiment has shown that PCOS induction in female rats resulted in significant increases as far as circulating androgen profile including androstendione, DHEAS and total testosterone as well as free testosterone versus control ones. According to research, these parameter elevations are commonly utilised as hyperandrogenaemia biomarkers in PCOS (Pinola et al., 2015). This suggests that PCOS is regarded as a disorder caused by increased androgen biosynthesis, usage or metabolism as reiterated by Azziz et al. (2006). Ovary is perceived as the principal androgens' source in majority PCOS patients. However, Kamel et al. (2005) and Baptiste et al. (2010) discovered that the adrenal androgens levels especially DHEAS had increased among more than half of PCOS patients' percentage. In a related experiment conducted by Kamel et al. (2005), there were signs of adrenocortical products' hypersecretion among the patients as they reacted to adrenocorticotropic hormone (ACTH) stimulation. Furthermore, this experiment showed that excess in adrenal androgen in PCOS appears so because of hyperplasia of adrenal cortex that has a concomitant hyperactivity of the adrenal gland. This leads to elevated DHEAS levels among such patients as noted in a similar research by Unlu et al. (2016). An increase in steroidogenic enzyme17b-hydroxysteroid dehydrogenase (17b-HSD) increase was also found in 
PCOS condition. It is noteworthy that the enzyme can catalyse the androstenedione conversion to testosterone, resulting in steroids over-generation, for example, testosterone, an observation made by Nelson et al. (1999). Additionally, the indication that the testosterone level in PCOS increased may be because the conversion of androgens to estradiol was blocked (Rezvanfar et al., 2012).

The findings of this investigation illustrated as well the presence of a positive co-relation between total testosterone, free testosterone and DHEAS in PCOS rats. The result thus gives a suggestion of DHEAS undergoing continuous inter-conversion as well as converting to testosterone and androstenedione through peripheral tissues (Bird et al., 1984; Unlu et al., 2016). To the contrary, Nestler (1991) provided evidence that exhibited hyperinsulinemia associating with PCOS and adding to increased androgen synthesis by ovary as well as reduced biosynthesis of sex hormone-binding globulin (SHBG) by hepatocytes, leading to high level of bioavailability of free androgens.

When administered, Vitex agnus-castus (Vit) fruit powder or metformin (Met) to rats induced with PCOS, markers showed a down-modulation with regard to the androgen profile increases that included androstendione, DHEAS and total testosterone as well as free testosterone in comparison to control ones. It became evident that Vit had more effectiveness concerning modulation of the circulating androgen profile. To the best of knowledge, this study can be regarded as the first investigation, which proved that Vit fruit has a hypoandrogenism impact on PCOS. Therefore, it may be suggested in this study that Vit friut's hypoandrogenic effect can relate with its active compound known as flavonoid. This investigation reports, as a result, that the presence of flavonoid in several plants has ability to inhibit testosterone secretion when they interfere with the effect of insulin such as growth facto-1 ( IGF1 ) on the ovarian stroma (Vijayababu et al., 2006). Furthermore, Ibanez et al (2000) realised that Met improved significantly free androgen marker, blood total testosterone and androstenedione, as well as DHEAS.

Hyperlipidemia is among the metabolic disorders which are linked to PCOS (Valkenburg et al., 2008) that is viewed to be a significant risk factor especially in the cardiovascular diseases' incidence. Therefore, the current study exhibited that inducing rats with PCOS made them to have hyperlipidemia and is the reason there is increased levels of circulation of total lipids as well as apo-B while apo A protein decreased concomitantly in PCOS induced rats compared to control ones. In the same manner, Ying et al. (2015) have reported alterations as far as the lipid parameters among PCOS induced patients. Apo A is perceived as the structural component which is principle in the high-density lipoprotein cholesterol (HDL-C) that can benefit during suppression of low-density lipoprotein cholesterol (LDL-C) oxidation. ApoB refers to the primary component of blood atherogenic lipoproteins, which includes LDL and intermediate density lipoprotein (IDL) as well as lipoprotein (VLDL) that is quite low in density. When apo A decreases and apo B increases the circulation of PCOS rats presented in the current study may result in atherogenic lipid profile (LDL-C, IDL and VLDL) increasing and antitherogenic HDL-C decreasing, with a decrease in the risk elements for atherogenesis as well as the cardiovascular events' development. This assertion is not only a revelation of the current study but also a confirmation by previous clinical practice (Dilbaz et al., 2011 ). The change with respect to lipid profile in PCOS may be due to the fact that the increase level of androgens that have a significant role in insulin receptor insensitivity, and which leads to insulin resistance as observed by Moranet al. (2010). The resistance originating from insulin stimulates triglycerides lypolysis to free fatty acid through peripheral tissues, which are then released into blood circulation. This leads to input of these fatty acid to liver which synthesises apoB as well as VLDL which finally causes hyperlipidemia (Valkenburg et al., 2008). Such a notion gets support from authors such as Baldani et al. (2012), who opine that hyperlipidemia in PCOS can be associated with insulin resistance. It is reiterated that Met or Vit fruit administration to rats induced with PCOS, markedly ameliorate the elevation in the circulating of total lipids and apo-B as well as leads to the depletion in apo A protein in comparison to PCOS untreated ones. It is true that Met or Vit's hypolipidemic effects are beneficial. This is widely documented in previous clinical researches conducted by Katakam et al. (2000) and Abu-Raghif et al. (2015). For instance, Abu-Raghif et al. (2015) demonstrated how Vit extracts minimised effectively the atherogenic lipid profile (TC, TG, LDL and VLDL) among rodents having hyperlipidemia, and which suggested that Vit extracts contain various components including flavonoids and diterpenoids as well as iridoids with effects of hypolipidemic. In addition, it has been established that hyperprebetalipoproteinemia patients' treatment using Met led to a marked depletion in blood triglycerides as well as cholesterol. Katakam et al. (2000) acknowledges that the effect of Met's hypolipidemic may be associated with its insulin sensitising action.

With regard to this experiment, the content of serum in homocysteine (Hcy) appeared significantly high in PCOS female rats compared with control ones. The finding concurs with a report done earlier which illustrated hyperhomocysteinemia as among the metabolic abnormalities in patients with PCOS (Esmaeilzadeh et al., 2017). Badawy et al. (2007) echoes that Hcy features a sulfur amino acid, synthesised by breaking down methionine. An investigation carried out by Badawy et al. (2007) has associated hyperhomocysteinemia in PCOS with the high insulin level acting as an inhibitor to activity of cystathionine b-synthase,resulting in increased serum homocysteine. Other researches such as Nervana et al. (2012) carried out studies to show proof of hyperandrogenism in PCOS patients affecting Hcy metabolism and leading to its elevation in the circulation. 
Hyperhomocysteinemia appears as a hazard element for cardiovascular event in PCOS patients as illustrated by Henry (2011). There are also other reports that support the potential role that homocysteine plays in vascular endothelial injury as well as dysfunction. Chen et al. (2015) is one of the many supporting reports. Other scholars posited that hyperhomocysteinemia is a atherogenesis's risk factor since it reduces endothelium's thrombo-resistance (Al- Gareeb et al., 2016). When Met or Vit is ingested in PCOS rats, the effect significantly ameliorates the deviation in serum level of Hcy compared to PCOS untreated rats. Vit fruit has proved beneficial in reducing serum Hcy level in rats having PCOS. However, Riahinejad et al. (2015)'s study reveals no previous report to authenticate such an impact and is the reason this investigation was carried out to demonstrate the reducing impact of Vit fruit on serum Hcy in PCOS condition, but the results pointed towards Met as the one having reducing effect on blood Hcy level in PCOS patients.

The current study attempted to expand the scope of its investigation to cover the serum levels of adipokines with respect to PCOS rats. As a result, it was shown that the existence of a leptin levels increase and adeponectin level decrease in PCOS induced rats as compared to control rats. This result is in agreement with another clinical investigation which found that PCOS patients have blood hyperleptinemia as well as hypoadiponectinemia unlike normal ones conducted by Chen et al. (2015). Another study conducted in 2017 by Polak and colleagues shows that insulin resistance linked to PCOS has a significant role regarding adipokine abnormality's causes due to the major role it plays in their secretions from the adipocytes. From this study it became evident that hyperleptinemia causes cardiovascular homeostasis with its ability to stimulate vascular inflammation and oxidative stress as well as vascular smooth muscle hypertrophy which may add to pathogenesis of atherosclerosis as well as coronary heart disease, an observation also made in Beltowski (2006). In addition, low adeponectin level can yield a fundamental role in cardiovascular events as it has principle influence in the stimulation of fatty acid oxidation as well as suppression of atherosclerosis. Similar interpretations were made in Meier and Gressner (2004). Ingesting Met or Vit to rats induced with PCOS, markedly modulated the alterations witnessed in levels of serum adipokine versus PCOS untreated animals. Again, Vit appeared more effective in ameliorating the serum content of adipokine. The finding confirms this study's significance since there has never been any published data regarding the modulating impact of Vitfruit on adepokines. This experiment attempted to suggest the beneficial impact of Vit fruit on adepkines and that such impact may attribute to its hypoandrogenism, but the results pointed to Met showing that it is its modulating impacts on leptin and adeponectin levels that may relate to its hypoandrogenism and activating effects on insulin receptors (Ismail et al., 2013).

It is also significant to mention another cardiovascular hazard element associated with PCOS known as Hyperprolactinemia (Yilmaz et al., 2015). The current study demonstrated that inducting PCOS in rats significantly led to the serum prolactin level increasing unlike control animals. Increased prolactin level circulation plays an important role in the incidence of atherosclerosis (Medic-Stojanoska et al., 2015). According to Sun et al. (2004) and Georgiopoulos et al. (2009), hyperprolactinemia promotes prolactin receptors' overexpression within the walls of coronary arteries, with a capability of stimulating the adhesion of monocyte-macrophage system to vascular endothelial cells as well as the vascular smooth muscle cell's proliferation resulting in atherosclerosis. Medic-Stojanoska et al. (2015) adds that clinical research has established that high level of serum LDL-C has a positive correlation with hyperprolactinemia. When PCOS rats are administered with either Vit or Met, the level of serum prolactin decreases in a significant way compared to PCOS untreated ones. Even in this case, Sliutz et al. (1993), Arentz et al. (2014) and Elsersy (2017) place forth an observation that Vit fruit treatment showed more benefits in restoring the serum prolactin to the normal level. The prolactin depleting impact of both Met and Vit has been documented. Vit, therefore, appeared to have a lowering effect on prolactin secretion, and this is due to its varying phyto compounds (Sliutz et al., 1993, Arentz et al., 2014).

The findings of the current study also revealed increased serum level of 8 -OHdG in PCOS induced rats against control animals. Elevated $8-\mathrm{OHdG}$ is among the marker of oxidative DNA lesions in response to oxidative stress in the view of Valavanidis et al. (2009). Previous clinical investigations carried out by Rezvanfar and colleagues (2012) confirm that hyperandrogenism in PCOS led to oxygen free radicals' production that majorly damage oxidative DNA. Such a result may give a perception that hyperandrogenism causes arrest in cell cycle and consequently cell death. Met or Vit administration to PCOS induced rats, significantly minimises the elevated serum $8-\mathrm{OHdG}$ compared with PCOS rats. This shows their antioxidant potential effects. Previous studies have confirmed the antioxidant impacts of Met as well as Vit (Ono et al., 2011; Jamilian et al., 2017). Ono et al. (2011) reiterate that this antioxidant impact of Vit plant has been attributed to the active component called flavonoid. The compound is portrayed by scholars to have scavenging effect on $\mathrm{O}_{2}^{-}$and $\mathrm{H} 2 \mathrm{O} 2$ as well as NO radicals that are extremely effective in oxidative DNA damage. Furthermore, other authors have found Met to have modulating impact against DNA oxidative lesion emanating from doxorubicin (Oliveira et al., 2017).

This investigation also showed increases in serum cardiac damage indices including troponin T, and LDH as well as ALP in PCOS rats unlike control animals. It is also important to note that the myocardial damage can 
attribute to the metabolic abnormalities that includes dyslipidemia, hyperhomocysteinemia, hyperleptinemia, hypoadiponectinemia and hyperprolactinemia as well as oxidative DNA damage brought by hyperandrogenism in PCOS female rats. These markers namely troponin T and LDH as well as ALP has been confirmed to be important in predicting the myocardial death or myocardial infarction (Hamm et al., 1992). Met or Vit ingestion pronouncedly reduced the elevated serum markers of myocardial injury in PCOS rats. This result may indicate the therapeutic potential of both Met and Vit against cardiac damage linked to metabolic alterations during PCOS condition.

\section{Conclusion:}

This paper reports a study which has demonstrated how Met and Vit fruit can be effectively utilised in the management of hyperandrogenism caused by PCOS. This is because both agents are capable of ameliorating markers of hyperandrogenism and related cardiovascular risk elements, including dyslipidemia, hyperhomocysteinemia, hyperleptinemia, hypoadiponectinemia and hyperprolactinemia as well as oxidative DNA damage. The experiment has established that Vit fruit powder is more successful in modulating most of these parameters to near normal levels than Met. These beneficial impacts of Vit plants may be as a result of its active compounds and is probably what supports its utilisation in the management of cardiovascular events related to PCOS.

\section{REFERENCES}

Abu-Raghif, A.R., H.B. Sahib, S.N. Abbas, 2015. Anti-hyperlipidemic effect of Vitex agnus castus Extracts in Mice. Int. J. Pharm. Sci. Rev. Res., 35(2)23: 120-125

Al- Gareeb, A.I., W.S. Abd Al- Amieer, H.M. Alkuraishy, T.J. Al- Mayahi, 2016. Effect of body weight on serum homocysteine level in patients with polycystic ovarian syndrome: A case control study. Int J Reprod BioMed, 14: 81-88.

Allahtavakoli, M., N. Honari, I. Pourabolli, M. Arababadi, H. Ghafarian, A. Roohbakhsh, A. Nadimi and A. Shamsizadeh, 2015. Vitex Agnus Castus Extract Improves Learning and Memory and Increases the Transcription of Estrogen Receptor $\alpha$ in Hippocampus of Ovariectomized Rats. Basic and clinical neuro science, 6(3): 185-192.

Arentz, S., J.A. Abbott, C.A. Smith, A. Bensoussan, 2014. Herbal medicine for the management of polycystic ovary syndrome (PCOS) and associated oligo/amenorrhoea and hyperandrogenism; a review of the laboratory evidence for effects with corroborative clinical findings. BMC Complement Altern Med., 14: 511.

Azziz, R., E. Carmina, D. Dewailly, E. Diamanti-Kandarakis, H.F. Escobar-Morreale, W. Futterweit et al., 2006. Positions statement: criteria for defining polycystic ovary syndrome as a predominantly hyperandrogenic syndrome: an Androgen Excess Society guideline. J Clin Endocrinol Metab., 91: 423-745.

Badawy, A., O. State, S. El Gawad, O.A. El Aziz, 2007. Plasma homocysteine and polycystic ovary syndrome: the missed link, Eur. J. Obstet Gynecol. Reprod. Biol., 131(1): 68-72.

Baldani, D.P., L. Skrgatić, M.S. Goldstajn, G. Zlopasa, S.K. Oguić, T. Canić, et al. 2012. Clinical and biochemical characteristics of polycystic ovary syndrome in Croatian population. Coll Antropol, 36: 1413-1418.

Baptiste, C.G., M.C. Battista, A. Trottier, J.P. Baillargeon, 2010. Insulin and hyperandrogenism in women with polycystic ovarysyndrome. Steroid Biochem Mol Biol., 122-1-21

Beltowski, J., 2006. Leptin and atherosclerosis. Atherosclerosis, 189: 47-60.

Bird, C.E., V. Masters, A.F. Clark, 1987. Dehydroepiandrosterone sulfate: kinetics of metabolism in normal young men and women. Clin Invest Med., 7: 119-22.

Chen, C.I., M.I. Hsu, S.H. Lin, Y.C. Chang, C.S Hsu, C.R. Tzeng, 2015. Adiponectin and leptin in overweight/obese and lean women with polycystic ovary syndrome. Gynecol Endocrinol., 31(4): 264-8.

Chen, H., Y. Sun, X. Wang, Q. Si, W. Yao, Z. Wan, 2015. Association of cardiometabolic risk profile with prehypertension accompany hyperhomocysteinaemia. Clin Exp Hypertens, 37: 218-222.

Coyotupa, J.A., F. Parlow and G.E. Abraham, 1973. Simultaneous radioimmunoassay of plasma testosterone and dihydrotestosterone. Anal. Lett., 5: 329-339.

Dilbaz, B., E. Ozkaya, M. Cinar, E. Cakir, S. Dilbaz, 2011. Cardiovascular disease risk characteristics of the main polycystic ovary syndrome phenotypes. Endocrine, 39: 272-277.

Ehrmann, D.A., 2005. Polycystic ovary syndrome. N Engl J Med., 352: 1223e36.

Elsersy, M.A.M., 2017. Efficacy of Combined Cabergoline and Metformin Compared to Metformin Alone on Cycle Regularity in Patients with Polycystic Ovarian Disease with Hyperprolactinemia: A Randomized Clinical Trial. J Obstet Gynaecol India, 67(5): 363-369.

Endoh, A., S.B. Kristiansen, P.R. Casson, J.E. Buster, P.J. Hornsby, 1996. The zona reticularis is the site of biosynthesis of dehydroepiandrosterone and dehydroepiandrosterone sulfate in the adult human adrenal cortex resulting from its low expression of 3-hydroxysteroid dehydrogenase. J Clin Endocrinol Metab, 81: 3558-3565. 
Esmaeilzadeh, S., E. Tahmasbpour, M.G. Chari, 2017. Hyperhomocysteinemia, insulin resistance and body mass index in Iranian young women with polycystic ovary syndrome. Middle East Fertility Society, pp: 149155.

Georgiopoulos, G.A., K.S. Stamatelopoulos, I. Lambrinoudaki, M. Lykka, K. Kyrkou, D. Rizos, M. Creatsa, G. Christodoulakos, M. Alevizaki, P.P. Sfikakis, C. Papamichael, 2009. Prolactin and preclinical atherosclerosis in menopausal women with cardiovascular risk factors. Hypertension, 54: 98-105.

Hamm, C.W., J. Ravkilde, W. Gerhardt, P. Jorgensen, E. Peheim, L. Ljungdahl et al., 1992. The prognostic value of serum troponin T in unstable angina.NEngl JMed, 327(3): 146-150.

Henry, O., 2011. Correlation between Homocysteine Levels and Risk Factors for Cardiovascular Disease: Age, Gender, and BMI Dependency among Jackson Heart Study Participants. The University of Mississippi Medical Center, 162: 3475971.

Ibanez, L., C. Valls, N. Potau, et al., 2000. Sensitization to insulin in adolescent girls to normalize hirsutism, hyperandrogenism, oligomenorrhea, dyslipidemia, and hyperinsulinism after precocious pubarche. $\mathrm{J}$ Clin Endocrinol Metab, 85: 3526-3530.

Ibrahim, N., A.S. Shalaby, R.S. Farag, G.S. Elbaroty, S.M. Nofal, E.M. Hassan, 2008. Gynecological efficacy and chemical investigation of Vitex agnus-castus L. fruits growing in Egypt. Nat Prod Res., 22(6): 537546.

Jang, M., M.J. Lee, J.M. Lee, C.S. Bae, S.H. Kim, J.H. Ryu et al., 2014. Oriental Medicine Kyung-Ok-Ko Prevents and Alleviates Dehydroepiandrosterone-Induced Polycystic Ovarian Syndrome in Rats. plos one. 9: 2.

Jamilian, H., M. Jamilian, F. Foroozanfard, F. Afshar Ebrahimi, F. Bahmani, Z. Asemi, 2017. Comparison of myo-inositol and metformin on mental health parameters and biomarkers of oxidative stress in women with polycystic ovary syndrome: a randomized, double-blind, placebo-controlled trial. J Psychosom Obstet Gynaecol., 5: 1-8.

Kamel, N., V. Tonyukuk, R. Emral, D. Corapc,io־glu, M. Bas stemir, S. Güllü, 2005. Role of ovary and adrenal glands in hyperandrogenemia in patients with polycystic ovary syndrome. Exp Clin Endocrinol Diabetes, 113: 115-21.

Ismail, T.A., M.M. Soliman, S.A. Ismail, 2013. Adiponectin regulation in type 2 diabeticrats: Effect of insulin, metfomin and dexamethasone. J Pharmacol Toxicol., 8: 197-208.

Katakam, P.V., M.R. Ujhelyi, M. Hoenig, A.W. Miller, 2000. Metformin improves vascular function in insulin-resistant rats. Hypertension., 35(1): 108e112.

Lal, J. and G. Jain, 2010. Effect of centchroman coadministration on the pharmacokinetics of metformin in rats. Indian J Pharmacol., 42(3): 146-149.

Lizneva, D., L. Gavrilova-Jordan, W. Walker, R. Azziz, 2016. Androgen excess: investigations and management. Best Pract Res Clin Obstet Gynaecol.

Medic-Stojanoska, M., T. Icin, I. Pletikosic, I. Bajkin, J. Novakovic-Paro, E. Stokic, D.T. Spasic, B. Kovacev-Zavisic, L. Abenavoli, 2015. Risk factors for accelerated atherosclerosis in young women with hyperprolactinemia. Med Hypotheses, 84(4): 321-6.

Meier, U., A.M. Gressner, 2004. Endocrine regulation of energy metabolism: review of pathobiochemical and clinical chemical aspects of leptin, ghrelin, adiponectin, and resistin. Clin Chem, 50: 151-11.

Moghetti, P., R. Castello, C. Negri, et al., 2000. Metformin effects on clinical features, endocrine and metabolic profiles, and insulin sensitivity in polycystic ovary syndrome: a randomized, double-blind, placebocontrolled 6-month trial, followed by open, long-term clinical evaluation. J Clin Endocrinol Metab, 85: 139-146.

Nakae, D., Y. Mizumoto, E. Kobayashi, O. Noguchi, Y. Konishi, 1995. Improved genomic/nuclear DNA extraction for 8-hydroxydeoxyguanosine analysis of small amounts of rat liver tissue. Cancer Lett., 97: 233-239.

Nelson, V.L., R.S. Legro, J.F. Strauss, et al., 1999. Augmented androgen production is a stable steroidogenic phenotype of propagated theca cells from polycystic ovaries. Mol Endocrinol., 13(6): $946 \mathrm{e} 57$.

Nestler, J.E., 1997. Role of hyperinsulinemia in the pathogenesis of the polycystic ovary syndrome, and its clinical implications. Semin Reprod Endocrinol., 15(2): 111-22.

Orio, F., S. Palomba, A. Colao, 2006. Cardiovascular risk in women with polycystic ovary syndrome. Fertil Steril., 86(Suppl. 1): S20-1.

Nervana, M.K., Bayoumy, Mohamed M. El-Shabrawi, K.A. Atwa, 2012. Assessment of homocysteine plasma levels and insulin resistance among obese women with anovulatory, Infertility. Life Sci. J. 9(4): 15991604.

Oliveira, V.C., S.A.R. Constante, P.C. Orsolin, J.C. Nepomuceno, A.A.A. de Rezende, M.A. Spanó, 2017. Modulatory effects of metformin on mutagenicity and epithelial tumor incidence in doxorubicin-treated Drosophila melanogaster. Food Chem Toxicol., 106: 283-291.

Ono, M., K. Eguchi, M. Konoshita, C. Furusawa, J. Sakamoto, S. Yasuda, T. Ikeda, M. Okawa, J. Kinjo, H. Yoshimitsu, T. Nohara, 2011. A new diterpenoid glucoside and two new diterpenoids from the fruit of Vitex agnus-castus. Chem Pharm Bull (Tokyo). 59(3): 392-6. 
Pinola, P., T.T. Piltonen, J. Puurunen, E. Vanky, I. Sundström-Poromaa, E. Stener-Victorin, K. Ruokonen, K. Puukka, J.S. Tapanainen, L.C. Morin-Papunen, 2015. Androgen profile through life in women with polycystic ovary syndrome: a nordic multicenter collaboration study. J. Clin. Endocrinol. Metab., 100: 34003407.

Polak, K., A. Czyzyk, T. Simoncini1, B. Meczekalski, 2017. New markers of insulin resistance in polycystic ovary syndrome. J Endocrinol Invest, 40: 1-8.

Riahinejad, S., A. Riahinejad, E. Alizadeh, 2015. Effects of Metformin Treatment on Homocysteine Levels and Metabolic Parameters of Women With Polycystic Ovary Syndrome. Journal of Family and Reproductive Health, 9: 172-176.

Reaven, G.M., P. Johnston, C.B. Hollenbeck, et al., 1992. Combined metformin-sulfonylurea treatment of patients with noninsulin-dependent diabetes in fair to poor glycemic control. J Clin Endocrinol Metab, 74: 10201026.

Reddy, S.P., N. Begum, S. Mutha and V. Bakshi, 2016. Beneficial effect of Curcumin in Letrozole induced polycystic ovary syndrome. Asian Pacific Journal of Reproduction, 5(2): 116-122.

Refsum, H., S. Hellimd, P.M. Uelalid, 1985. Radioenzymje determination of homocyateine in plasma and urine. Clin Chem, 31: 824-828.

Rezvanfar, M.A., M.A. Rezvanfar, A. Ahmadi, H.A. Shojaei-Saadi, M. Baeeri, M. Abdollahi, 2012. Molecular mechanisms of a novel selenium-based complementary medicine which confers protection against hyperandrogenisminduced polycystic ovary. Theriogenology, 78(3): 620-31.

Ren, M.Q., G. Kuhn, J. Wegner, J. Chen, 2001. Isoflavones, substances with multi-biological and clinical properties. Eur J Nutr., 40(4): 135-146.

Sliutz, G., P. Speiser, A.M. Schultz, J. Spona, R. Zeillinger,1993. Agnus-castus extracts inhibit prolactin secretion of rat pituitary cells. Horm Metab Res., 25(5): 253-255.

Sun, R., A.L. Li, H.M. Wei, Z.G. Tian, 2004. Expression of prolactin receptor and response to prolactin stimulation of human NK cell lines. Cell Res., 14: 67-73.

Taher, M.A., Y.A. Atia, M.K. Amin, 2012. Improving an Ovulation Rate in Women with Polycystic Ovary Syndrome by Using Silymarin. Global Journals Inc., 12(6): 16-21.

Unlu, E., B.S. Unlu, Y. Yildiz, M. Beker-Acay, E. Kacar, O. Turamanlar, O.B. Tulmac, A. Seven, U. Ozuguz, 2016. Adrenal gland volume assessed by magnetic resonance imaging in women with polycystic ovary syndrome. Diagnostic and Interventional Imaging, 97: 57-63.

Valavanidis, A., T. Vlachogianni, C. Fiotakis, 2009. 8-hydroxy-2' -deoxyguanosine (8-OHdG): A critical biomarker of oxidative stress and carcinogenesis. J Environ Sci Health C Environ Carcinog Ecotoxicol Rev., 27(2): 120-39.

Valkenburg, O., R.P. Steegers-Theunissen, H.P. Smedts, G.M. Dallinga-Thie, B.C. Fauser, E.H. Westerveld, et al., 2008. A more atherogenic serum lipoprotein profile is present in women with polycystic ovary syndrome: a case-control study. J Clin Endocrinol Metab., 93: 470-476.

Vijayababu, M.R., A. Arunkumar, P. Kanagaraj and J. Arunakaran, 2006. Effects of quercetin on insulinlike growth factors (IGFs) and their binding protein-3 (IGFBP-3) secretion and induction of apoptosis in human prostate cancer cells . J Carcinog, 5: 10.

Weiss, R., 1988. Herbal Medicine. Gothenburg, Sweden: Beaconsfield Publishers, pp: 317-318.

Wuttke, W., H. Jarry, V. Christoffel, et al. 2003. Chaste tree (Vitex agnus-castus) - pharmacology and clinical indications. Phytomedicine, 10: 348-357.

Yarali, H., A. Yildirir, F. Aybar, G. Kabakci, O. Bukulmez, E. Akgul, et al., 2001. Diastolic dysfunction and increased serum homocysteine concentrations may contribute to increased cardiovascular risk in patients with polycystic ovarysyndrome, Fertil. Steril., 76(3): 511-516.

Yilmaz, Ö., M. Calan, T. Kume, M. Temur, P. Yesil, M.Y. Senses, 2015. The effect of prolactin levels on MPV in women with PCOS. Clin Endocrinol, 82(5): 747-52.

Ying, W.A.N.G., L.I. Yan, X.U. Fang, L.I. Yan-qiu, W.U. Jing, W.A.N.G. Gui-yan, L.I.U. Song-jiang, H.O.U. Li-hui, 2015. Apolipoprotein B/Apolipoprotein A1 ratio is associated with sex hormone binding globulin and may be an indicator of metabolic syndrome in PCOS women. Journal of Reproduction \& Contraception, 26(4): 213-221. 